\title{
Alena Vanžurová \\ On reducibility of double linear connections on a double vector fibration with soldering
}

Czechoslovak Mathematical Journal, Vol. 42 (1992), No. 2, 199-210

Persistent URL: http://dml.cz/dmlcz/128339

\section{Terms of use:}

(C) Institute of Mathematics AS CR, 1992

Institute of Mathematics of the Czech Academy of Sciences provides access to digitized documents strictly for personal use. Each copy of any part of this document must contain these Terms of use.

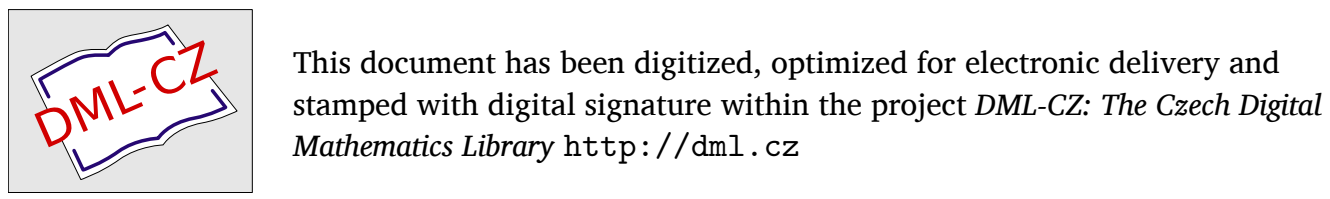




\title{
ON REDUCIBILITY OF DOUBLE LINEAR CONNECTIONS ON A DOUBLE VECTOR FIBRATION WITH SOLDERING
}

\author{
Alena Vanžurová, Olomouc
}

(Received September 24, 1990)

In this paper we will answer some questions about reducibility of connections on the principal fibrations of double linear frames corresponding to TTM and $T T^{*} M$ using the terminology introduced in $[10]-[11]$. The original concept of the category of double vector fibrations and morphisms is due to J. Pradines, [6], [7], and was developed by I. Kolář, [2]. Double linear connections were studied in [11], the isomorphisms called solderings were introduced in [2], [11], [12].

Under a (generalized) connection on a fibred manifold $\pi: Y \rightarrow M$ we understand a smooth section $\Gamma: Y \rightarrow J^{1} Y$ of the natural projection $\varrho_{0}^{1}: J^{1} Y \rightarrow Y$ on a target, $\varrho_{0}^{1} \circ \Gamma=$ id. If $\mathscr{C}, p: \mathscr{C} \rightarrow M$ is a double linear $(\mathscr{D} \mathscr{L}-)$ fibration with the underlying vector fibrations $\mathscr{A}, \mathscr{B}, \mathscr{Y}$, then $J^{1} \mathscr{C}$ (and more generally, $J^{r} \mathscr{C}$ for $r \geqq 0$ ) is also endowed with a structure of a $\mathscr{D} \mathscr{L}$-fibration, the natural projection $\varrho_{0}^{1}: J^{1} \mathscr{C} \rightarrow \mathscr{C}$ (or $\varrho_{r}^{s}: J^{s} \mathscr{C} \rightarrow J^{r} \mathscr{C}$ ) being a morphism of $\mathscr{D} \mathscr{L}$-fibrations. A connection $\Gamma: \mathscr{C} \rightarrow J^{1} \mathscr{C}$ which is at the same time a double linear morphism of $\mathscr{D} \mathscr{L}$-fibrations, will be called a $\mathscr{D} \mathscr{L}$-connection. Any $\mathscr{D} \mathscr{L}$-connection, as a $\mathscr{D} \mathscr{L} \mathscr{F}$-morphism, induces three underlying linear connections $\Gamma_{1}: \mathscr{A} \rightarrow J^{1} \mathscr{A}, \Gamma_{2} \mathscr{B} \rightarrow J^{1} \mathscr{B}$, and $\Gamma_{3}: \mathscr{Y} \rightarrow J^{1} \mathscr{Y}$. Similarly to the linear case, any $\mathscr{D} \mathscr{L}$-fibration is associated with a principal fibration of all double linear $(\mathscr{D} \mathscr{L}-)$ frames, denoted here by $\mathscr{F}$. A $\mathscr{D} \mathscr{L}$-frame on $\mathscr{C}$, at a point $x$, is a $\mathscr{L} \mathscr{L}$-isomorphism $f: K(n, s, t) \rightarrow \mathscr{C}_{x}$ of the trivial $\mathscr{D} \mathscr{L}$-space $K(n, s, t)=$ $=\mathbb{B}^{n} \times \mathbb{R}^{s} \times \mathbb{P}^{t}$ onto the fibre $\mathscr{C}_{x}$ through $x \in M$. The structure group Aut $(n, s, t)$ of $\widetilde{F}$ is the group of all $\mathscr{D} \mathscr{L}$-automorphisms of the trivial $\mathscr{D} \mathscr{L}$-space $K(n, s, t)$. The associated fibration $\mathscr{F}(K(n, s, t))$ is $\mathscr{D} \mathscr{L} \mathscr{F}$-isomorphic to $\mathscr{C}$.

On the principal fibration $\mathscr{F}$, we admit ,principal" connections only, i.e. connections $\Delta$ satisfying the right invariant property $\Delta(f . g)=\Delta(f) . g$ for any frame $f \in \mathscr{F}$ and any element $g$ of the structure group.

The results obtained here are motivated by the following consideration. The second tangent and cotangent spaces $T T M, T T^{*} M, T^{*} T M$, and $T^{*} T^{*} M$ can be regarded as soldered $\mathscr{D} \mathscr{L}$-fibrations, all associated with the principal fibration $H^{2} M$ of second order frames on $M$, its structure group being $L_{m}^{2}$. Since the functors $T^{*} T$ and $T^{*} T^{*}$ are naturally equivalent to $T T^{*}$, we can omit the last two cases.

The $\mathscr{D} \mathscr{L}$-fibration $T T M$ has the underlying vector fibrations $\mathscr{A}=\mathscr{B}=\mathscr{V}=$ 
$=\left(T M, p_{M}, M\right)$, the $T T$-solderings $X_{1}, X_{2}: T M \rightarrow T M$ being $X_{1}=X_{2}=\mathrm{id}_{T M}$. Any principal invariant connection $\Delta$ on $H^{2} M$ induces a generalized connection on TTM, denoted by $\Gamma=T T(\Delta): T T M \rightarrow J^{1} T T M$. Now we can ask when a connection $\Gamma$ on $T T M$ is of the form $\operatorname{TT}(\Delta)$ for any invariant connection $\Delta$ on $H^{2} M$. This problem was, in a slightly modified version, solved in [8].

It can be verified that $T T(\Delta)$ is a $\mathscr{D} \mathscr{L}$-connection. Hence double-linearity is a necessary condition for $\Gamma$ to be of the above form. Further, we will describe a monomorphism $h$ of $H^{2} M$ into the principal subfibration $\mathscr{F}_{s}$ of $\mathscr{F}$, containing so called soldered frames, and characterize the image $\mathscr{F}_{s s}=h\left(H^{2} M\right)$ by vanishing of the ,structure function" introduced on $\mathscr{F}_{s}$. Now any connection on $H^{2} M$ is, in fact, a connection on $\mathscr{F}_{s s}$, and can be extended to a connection $\Delta^{\prime}$ on $\mathscr{F}_{s}$, and to $\Delta^{\prime \prime}$ on $\mathscr{F}$. Since there is an isomorphism ‘ associating any $\mathscr{D} \mathscr{L}$-connection $\Gamma$ on a $\mathscr{D} \mathscr{L}$ fibration with an invariant connection $\iota \Gamma$ on the principal fibration of $\mathscr{P} \mathscr{L}$-frames, we can write $\Delta^{\prime \prime}=\iota \Lambda$ for a unique $\mathscr{D} \mathscr{L}$-connection $\Lambda$ on TTM. By Theorem 1 , the underlying linear connections of $\iota \Lambda$ satisfy $\iota \Lambda_{1}=\iota \Lambda_{2}=\iota \Lambda_{3}$, since $\iota \Lambda=\Delta^{\prime \prime}$ is reducible to $\mathscr{F}_{s}$, and the maps tangent to solderings are $T X_{1}=T X_{2}=\mathrm{id}_{T T M}$. Consequently, we obtain a condition

$$
\Lambda_{1}=\Lambda_{2}=\Lambda_{3}
$$

for the underlying connections of $\Lambda$. Finally, by Theorem 6 , the reducibility of $\Delta^{\prime \prime}$ to the principal subfibration $\mathscr{F}_{s s}$ is equivalent to the $i$-invariance of $\Lambda$ with respect to the canonical involution $i$ on TTM, $J^{1}\left(i^{-1}\right) \circ \Lambda \circ i=\Lambda$. Together, we can give the following answer: $\Gamma=T T(\Delta)$ if and only if $\Gamma$ is double linear, $i$-invariant, and the underlying linear connections coincide.

In the paper, similar statements for $T T^{*}$-soldered $\mathscr{D} \mathscr{L}$-spaces are deduced. Similarly, $T^{*}(\Delta)$ is a $\mathscr{D L}$-connection, and Theorems 2, 10 describe the situation. Let us remark that there is no "canonical" involution on $T T^{*} M$. To characterize reducibility, we use an isomorphism between $\mathscr{F}_{s}$ and the principal fibration $\widetilde{\mathscr{F}}_{s}$ of $T T^{*}$-soldered $\mathscr{D} \mathscr{L}$-frames on $T T^{*} M$.

\section{PRELIMINARIES}

Let $C$ denote a double vector space ( $\mathscr{D} \mathscr{L}$-space) over reals with the natural projection $\pi: C \rightarrow A \times B$ and with the centre (kernel) $V,[10]$. If $\operatorname{dim} A=n, \operatorname{dim} B=s$, $\operatorname{dim} V=t$ we set $\operatorname{dim} C=(n, s, t)$. Any two double vector spaces are $\mathscr{D} \mathscr{L}$-isomorphic iff they have the same dimension. Hence $C$ is isomorphic to the trivial $\mathscr{D} \mathscr{L}$-space $K(n, s, t)=\mathbb{R}^{n} \times \mathbb{R}^{s} \times \mathbb{R}^{t}$ with the natural projection $K \rightarrow \mathbb{R}^{n} \times \mathbb{R}^{s}$ and centre $\mathbb{R}^{t}$. A $\mathscr{D} \mathscr{L}$-frame in $C$ is a $\mathscr{D} \mathscr{L}$-isomorphism $f: K(n, s, t) \rightarrow C$, the set $F(C)$ of all frames in $C$ forms a Lie group diffeomorphic with the Lie group $\operatorname{Aut}(n, s, t)$ of all $\mathscr{D} \mathscr{L}$-automorphisms of $K(n, s, t),[11]$. Any frame $f$ in $C$ determines linear isomorphisms $\tau_{1} f=f_{1}: \mathbb{R}^{n} \rightarrow A, \tau_{2} f=f_{2}: \mathbb{R}^{s} \rightarrow B$, and $\tau_{3} f=f \mid \mathbb{R}^{t} \rightarrow V$, i.e. frames in $A, B$, and $V$, respectively. 
Let $(\mathscr{C}, p, M)$ be a double vector fibration, [11], with the underlying vector fibrations $\left(\mathscr{A}, p_{1}, M\right),\left(\mathscr{B}, p_{2}, M\right),\left(\mathscr{Y}^{\circ}, p_{3}, M\right)$. As in the case of vector fibrations (bundles), there is a principal fibration of double linear frames associated with $\mathscr{E}$. The union $\mathscr{F}=\bigcup_{x \in M} F\left(\mathscr{C}_{x}\right)$ of all $\mathscr{D} \mathscr{L}$-frames (on fibres $\mathscr{C}_{x}$ of $\mathscr{C}$ over $x \in M$ ) forms a principal fibration $(\mathscr{F}, q . M)$ over $M$ with the structure group $\operatorname{Aut}(n, s, t)$ and projection $q: \mathscr{F} \rightarrow M, q(f)=x$ where $x$ is such an element of $M$ that $f \in F\left(\mathscr{C}_{x}\right)$. Any frame $f \in \mathscr{C}_{x}$ determines elements $\tau_{1} f, \tau_{2} f, \tau_{3} f$ which can be regarded as elements of the fibres $\mathscr{\mathscr { F }}_{1, x}, \mathscr{F}_{2, x}, \mathscr{F}_{3, x}$ of the principal fibrations $\left(\mathscr{F}_{1}, q_{1}, M\right.$, Aut $\left.(n)\right),\left(\mathscr{F}_{2}, q_{2}, M\right.$, $\operatorname{Aut}(s)),\left(\mathscr{F}_{3}, q_{3}, M, \operatorname{Aut}(t)\right)$ corresponding to the underlying vector fibrations $\mathscr{A}, \mathscr{B}, \mathfrak{Y}$ of $\mathscr{C}$. In this way, we obtain smooth morphisms of principal fibrations over homomorphisms of structure groups

$$
\begin{aligned}
& \tau_{1}:(\mathscr{F} . q, M, \operatorname{Aut}(n, s . t)) \rightarrow\left(\mathscr{F}_{1}, q_{1}, M, \operatorname{Aut}(n)\right) \text { over } \\
& \operatorname{Aut}(n, s, t) \rightarrow \operatorname{Aut}(n),
\end{aligned}
$$

and similarly for $\tau_{2}: \mathscr{F} \rightarrow \mathscr{F}_{2}$ and $\tau_{3}: \mathscr{F} \rightarrow \mathscr{F}_{3}$. The morphisms $\tau_{1}, \tau_{2}, \tau_{3}$ determine a morphism of principal fibrations

$$
\tau=\left(\tau_{1}, \tau_{2}, \tau_{3}\right): \mathscr{F} \rightarrow(\tilde{\mathscr{F}}, q, M, \operatorname{Aut}(n) \times \operatorname{Aut}(s) \times \operatorname{Aut}(t))
$$

where $\widetilde{\mathscr{F}}=\mathscr{F}_{1} \times_{M} \mathscr{F}_{2} \times_{M} \mathscr{F}_{3}$ denotes the Whitney sum.

A $T T$-soldering (or $T T^{*}$-soldering) on the $\mathscr{L} \mathscr{L}$-space $C$ is a couple of linear isomorphisms

$$
\chi_{1}: V \rightarrow A, \quad \chi_{2}: V \rightarrow B
$$

(or $\chi_{1}: V-A \cdot \chi_{2}: V \rightarrow B^{*}$, respectively), [12]

A double linear morphism $\varphi=\left(\varphi_{1}, \varphi_{2}, \varphi_{3}, \sigma\right): C \rightarrow C^{\prime}$ of two $T T$-soldered (or $T T^{*}$-soldered) $\mathscr{D} \mathscr{L}$-spaces is called $T T$ - $\left(T T^{*}-\right)$ soldered, [11], [12], if the underlying linear morphisms $\varphi_{1}: A \rightarrow A^{\prime}, \varphi_{2}: B \rightarrow B^{\prime}, \varphi_{3}: V \rightarrow V^{\prime}$ satisfy

and

$$
\chi_{1}^{\prime} \varphi_{3}=\varphi_{1} \chi_{1}
$$

$$
\chi_{2}^{\prime} \varphi_{3}=\varphi_{2} \%_{2} \quad \text { (or } \varphi_{2}^{*} \chi_{2}^{\prime} \varphi_{3}=\chi_{2} \text {, respectively) } .
$$

A frame $f$ in the $T T$-soldered (or $T T^{*}$-soldered) $\mathscr{D} \mathscr{L}$-space $C$ is $T T$-(or $T T^{*}$-)soldered if

and

$$
\chi_{1} \tau_{3} f=\tau_{1} f
$$

$$
\chi_{2} \tau_{3} f=\tau_{2} f \quad \text { (or } \chi_{2} \tau_{3} f=\left(\tau_{2} f\right)^{*}, \text { respectively). }
$$

A $\mathscr{L} \mathscr{L}$-fibration $(\mathscr{C}, p, M)$ is $T T$-(or $T T^{*}$-) soldered if there exists a $\mathscr{L} \mathscr{L}$-space $C$ with $T T$ - $\left(T T^{*}-\right)$ soldering such that any point $x$ of $M$ has a neighborhood $U$ such that the restriction $\left(\mathscr{C}_{U}, p_{U}, M\right)$ of $\mathscr{C}$ to $U$ is isomorphic with $\left(U \times C, \operatorname{pr}_{1}, U\right)$ over identity. Any $T T$ - (or $T T^{*}$-) soldering on $\mathscr{C}$ induces, via linear isomorphisms

$$
\begin{aligned}
& \chi_{1, x}: \mathscr{Y}_{x} \rightarrow \mathscr{A}_{x}, \\
& \chi_{2, x}: \mathscr{Y}_{x} \rightarrow \mathscr{B}_{x} \quad\left(\text { or } \chi_{2, x}: \mathscr{V}_{x} \rightarrow \mathscr{B}_{x}^{*}\right),
\end{aligned}
$$


the isomorphisms of the underlying fibrations, [11],

$$
\begin{aligned}
& X_{1}:\left(\mathscr{V}, p_{3}, M\right) \rightarrow\left(\mathscr{A}, p_{1}, M\right), \\
& X_{2}:\left(\mathscr{V}, p_{3}, M\right) \rightarrow\left(\mathscr{B}, p_{2}, M\right) \quad\left(\text { or } X_{2}:\left(\mathscr{V}^{*}, p_{3}, M\right) \rightarrow\left(\mathscr{B}^{*}, p_{2}^{*}, M\right)\right) .
\end{aligned}
$$

\section{THE CONNECTIONS ON $T T$ - AND $T T^{*}$-SOLDERED $\mathscr{D} \mathscr{L}$-FIBRATIONS}

Consider a $\mathscr{D} \mathscr{L}$-fibration $\mathscr{C}$ with a $T T$-soldering, and assume a double linear connection $\Gamma: \mathscr{C} \rightarrow J^{1} \mathscr{C}$ on $\mathscr{C},[11]$, with the underlying linear connections $\Gamma_{1}$ on $\mathscr{A}$, $\Gamma_{2}$ on $\mathscr{B}$, and $\Gamma_{3}$ on $\mathscr{H}$. The set of all $T T$-soldered frames on $\mathscr{C}$ forms a principal fibration $\left(\overline{\mathcal{F}}_{s}, q_{s}, M\right), q_{s}=q / \mathscr{F}_{s}$, a subfibration of $(\mathscr{F}, q, M)$. The structure group of $\mathscr{F}_{s}$ is the group $\operatorname{Aut}_{s}\left(\mathbb{R}^{m} \times \mathbb{R}^{m} \times \mathbb{R}^{m}\right)$ of all $T T$-soldered $\mathscr{D} \mathscr{L}$-automorphisms of the trivial $\mathscr{I} \mathscr{L}$-space $\mathbb{P}^{3 m}$ with the canonical $T T$-soldering $\gamma_{1}=\%_{2}=$ id, $m=\operatorname{dim} M$.

Denote by $\mathscr{T}$ the set

$$
\tilde{\mathcal{T}}=\left\{\left(f_{1}, f_{2}, f_{3}\right) \in \tilde{\mathscr{F}} ; X_{1} f_{3}=f_{1}, X_{2} f_{3}=f_{2}\right\} .
$$

$\mathscr{T}$ is a closed submanifold in $\tilde{\mathscr{F}}$, and the following is satisfied:

Lemma 1. $f \in \mathscr{F}_{s}$ if and only if $\tau f=\mathscr{T}$.

Similarly as in the linear case, there is a one-to-one map between the set of double linear connections on $\mathscr{C}$ and the set of right invariant connections on the principal fibration $\mathscr{F}$. In both linear and double linear cases, let us denote this map by $\iota$. Now a natural question arises under what conditions the invariant connection $\iota \Gamma$ on $\mathscr{F}$ corresponding to $\Gamma$ on $\mathscr{C}$ can be reduced to $\mathscr{F}_{s}$.

Theorem 1. The invariant connection $[\Gamma$ is reducible to the principal subfibration $\left(\bar{F}_{s}, q_{s}, M\right)$ if and only if the horizontal subspaces $H_{1}, H_{2}$, and $H_{3}$ of connections ${ } \Gamma_{1},{ }_{1} \Gamma_{2}$, and $\iota \Gamma_{3}$ satisfy

$$
\left(T X_{1}\right) H_{3}=H_{1}, \quad\left(T X_{2}\right) H_{3}=H_{2} \text {. }
$$

Proof. (a) Suppose that $\iota \Gamma$ is reducible to $\mathscr{F}_{5}$. Let $f_{3} \in \mathscr{F}_{3}$, and let $\iota_{3} \in\left(H_{3}\right)_{f_{2}}$ be any element of the horizontal space of $\iota_{3} \Gamma_{3}$ at the point $f_{3}$. Define $f_{1}=X_{1} f_{3}$, $f_{2}=X_{2} f_{3}$, and choose $f \in \mathscr{F}$ so that $f=\left(f_{1}, f_{2}, f_{3}\right)$. Then $f \in \mathscr{F}_{s}$. In the horizontal space $H_{f}$ with respect to $\Gamma$, assume any vector $v \in H_{f}$ with the property $\left(T \tau_{3}\right) v=v_{3}$. Choose an $\iota \Gamma$-horizontal curve $\gamma:(-\varepsilon, \varepsilon) \rightarrow \mathscr{F}$ such that

$$
\gamma(0)=f, \quad \frac{\mathrm{d} \gamma(0)}{\mathrm{d} t}=v .
$$

Since $\gamma(0)=f \in \mathscr{F}_{s}$ and $\iota \Gamma$ is reducible to $\mathscr{F}_{s}$ we have $\gamma(t) \in \mathscr{F}_{s}$ for all $t \in(-\varepsilon, \varepsilon)$. By Lemma $1, \tau \gamma(t) \in \mathscr{T}$ for $t \in(-\varepsilon, \varepsilon)$, which means

$$
X_{1} \tau_{3} \gamma(t)=\tau_{1} \gamma(t), \quad X_{2} \tau_{3} \gamma(t)=\tau_{2} \gamma(t)
$$

for $t \in(-\varepsilon, \varepsilon)$. This implies

$$
\left(T X_{1}\right) v_{3}=v_{1}, \quad\left(T X_{2}\right) v_{3}=v_{2}
$$


where

$$
v_{1}=\frac{\mathrm{d}\left(\tau_{1} \gamma(0)\right)}{\mathrm{d} t} \in H_{1}, \quad v_{2}=\frac{\mathrm{d}\left(\tau_{2} \gamma(0)\right)}{\mathrm{d} t} \in H_{2} .
$$

This proves (1).

(b) Conversely, let (1) be fulfilled. Let $f \in \mathscr{F}_{s}$, and let $\gamma:(-\varepsilon, \varepsilon) \rightarrow \mathscr{F}$ be a horizontal curve of $\iota \Gamma$. Denote $f_{i}=\tau_{i} f, i=1,2,3$. The curve $\tau_{1} \gamma$ is a horizontal lift of the curve $q_{i} ;$ with respect to $\iota_{1} \Gamma_{1}$ through the point $\tau_{1} \gamma(0)=f_{1}$. Similarly, $\tau_{3} f$ is a horizontal lift of $q \gamma$ with respect to $\iota \Gamma_{3}$ through the point $\tau_{3} \gamma(0)=f_{3}$. But $X_{1} f_{3}=$ $=f_{1}$, and $\left(T X_{1}\right) H_{3}=H_{1}$. That is why $X_{1} \tau_{3}$ is also a horizontal lift of $q \gamma$ through $f_{1}$ with respect to $\iota \Gamma_{1}$. We obtain $X_{1} \tau_{3} \gamma(t)=\tau_{1} \gamma(t)$ for $t \in(-\varepsilon, \varepsilon)$. In a similar way, $X_{2} \tau_{3} \gamma(t)=\tau_{2} \gamma(t)$ for $t \in(-\varepsilon, \varepsilon)$. Thus $\tau \gamma(t) \in \mathscr{T}$ for all $t \in(-\varepsilon, \varepsilon)$, and $\gamma(t) \in \mathscr{F}_{s}$ for $t \in(-\varepsilon, \varepsilon)$. Therefore $\iota \Gamma$ is reducible to $\mathscr{F}_{s}$.

Now consider a $\mathscr{D} \mathscr{L}$-fibration $\mathscr{C}$ on $M$ with $T T^{*}$-soldering given by

$$
X_{1}:\left(\mathscr{H}^{*}, p_{3}, M\right) \rightarrow\left(\mathscr{A}, p_{1}, M\right), \quad X_{2}:\left(y^{*}, p_{3}, M\right) \rightarrow\left(\mathscr{B}^{*}, p_{2}^{*}, M\right) .
$$

All $T T^{*}$-soldered frames in $\mathscr{F}$ corresponding to $\mathscr{C}$ constitute again a principal fibration $\mathscr{F}_{s}$, a subfibration of $\mathscr{F}$. Its structure group is the group $\operatorname{Aut}_{s}\left(\mathbb{R}^{m *} \times\right.$ $\left.\times \mathbb{R}^{m} \times \mathbb{R}^{m *}\right)$ of all $T T^{*}$-soldered $\mathscr{D} \mathscr{L}$-automorphisms of the space $\mathbb{R}^{m *} \times \mathbb{R}^{m} \times$ $\times \mathbb{R}^{m *}$ with its canonical $T T^{*}$-soldering $\chi_{1}=$ id, $\chi_{2}=$ id.

The map $\tau: \mathscr{F} \rightarrow \widetilde{\mathscr{F}}=\mathscr{F}_{1} \times \mathscr{F}_{2} \times \mathscr{F}_{3}$ is again a surjective submersion. Let us define a closed submanifold $\mathscr{T}^{*}$ in $\widetilde{\mathscr{F}}$ by

$$
\mathscr{T}^{*}=\left\{\left(f_{1}, f_{2}, f_{3}\right) \in \widetilde{\mathscr{F}}, \quad X_{1} f_{3}=f_{1}, \quad X_{2} f_{3}=f_{2}^{*}\right\} .
$$

The frame $f$ in $\mathscr{F}$ is soldered iff $\tau f$ belongs to $\mathscr{T}^{*}$. Let $\Gamma, \Gamma_{i}, \mathscr{F}_{i}, i=1,2,3$, and $\iota \Gamma$ be as above. Denote by $\mathscr{F}_{2}^{*}$ the principal fibration corresponding to the vector fibration $\mathscr{B}^{*}$. A map associating any frame with its dual coframe gives an isomorphism $\mathscr{F}_{2} \rightarrow \mathscr{F}_{2}^{*}$ of principal fibrations over $M$. This isomorphism maps the invariant connection $\iota \Gamma_{2}$ on $\mathscr{F}_{2}$ onto an invariant connection $\iota \Gamma_{2}^{*}$ on $\mathscr{F}_{2}^{*}$.

Theorem 2. The right invariant connection " $\Gamma$ on the $T T^{*}$-soldered $D \mathscr{L}$-fibration' 6 is reducible to the subfibration $\mathscr{F}_{s}$ of $T T^{*}$-soldered frames if and only if the horizontal spaces $H_{1}, H_{2}^{*}, H_{3}$ of the connections $\iota \Gamma_{1}, \iota \Gamma_{2}^{*}, \iota \Gamma_{3}$ satisfy

$$
\left(T X_{1}\right) H_{3}=H_{1}, \quad\left(T X_{2}\right) H_{3}=H_{2} \text {. }
$$

The proof is similar as in the case of Theorem 1 .

\section{THE STRUCTURE FUNCTION AND REDUCTIONS}

Given an $m$-dimensional manifold $M$, let $H^{2} M=\operatorname{inv} J_{0}^{2}\left(\mathbb{R}^{m}, M\right)$ denote the principal fibration of second order frames on $M$ with the structure group $L_{m}^{2}$, the group of all invertible 2-jets on $\mathbb{R}^{m}$ with source and target $0, L_{m}^{2}=\operatorname{inv} J_{0}^{2}\left(\mathbb{R}^{m}, \mathbb{R}^{m}\right)_{0}$. This structure group can be regarded as a semidirect product of the linear group 
$L_{m}^{1}=G L(m, \mathbb{R})$ and the abelian group of all symmetric bilinear maps $\mathbb{R}^{m} \times \mathbb{R}^{m} \rightarrow$ $\rightarrow \mathbb{R}^{m}, L_{m}^{2}=L_{m}^{1} \times \operatorname{Hom}_{\text {sym }}\left(\mathbb{R}^{m} \times \mathbb{R}^{m}, \mathbb{R}^{m}\right),[10]$. So we can write its elements as couples $(\varphi, \sigma)$ with $\varphi \in L_{m}^{1}$ and $\sigma$ a symmetric bilinear homomorphism on $\mathbb{R}^{m}$. Furthermore, $L_{m}^{2}$ is isomorphic with the group $G_{s s}=A_{u t}\left(T T_{0} \mathbb{R}^{m}\right)$ of all strongly soldered automorphisms of the $\mathscr{D} \mathscr{L}$-space $T T_{0} \mathbb{R}^{m},[12]$, via the map

$$
\varkappa: L_{m}^{2} \rightarrow \operatorname{Aut}_{s s}\left(T T_{0} \mathbb{R}^{m}\right), \quad \varkappa(\varphi, \sigma)=(\varphi, \varphi, \varphi, \sigma) .
$$

We shall identify the both groups.

Consider now the principal fibration $\mathscr{F}$ or $\widetilde{F}_{s}$, of frames or $T T$-soldered frames, respectively, on TTM. We shall construct a morphism

$$
h: H^{2} M \rightarrow \mathscr{F}
$$

of principal fibrations as follows. An element $\varrho \in H_{x}^{2} M$ is of the form $\varrho=j_{0}^{2} \chi$ where $\alpha: U \subset \mathbb{R}^{m} \rightarrow M$ is a local diffeomorphism

$$
T T_{0} \alpha: T T_{0} \mathbb{R}^{m} \rightarrow T T_{x} M,
$$

a restriction of the map $T T \alpha$ to the fibre of $T T \mathbb{R}^{m}$ through the origin $0 \in \mathbb{R}^{m}$. This definition is independent of the choice of a diffeomorphism $\chi$ with the property $\varrho=j_{0}^{2} \alpha$. Since $T T_{0} \alpha$ respects the natural $T T$-solderings on $T T_{0} \mathbb{R}^{m}$ and $T T_{x} M$ we have $h(\varrho) \in \mathscr{F}_{s}$ for any $\varrho \in H^{2} M$. Hence we obtain a monomorphism of principal fibrations

$$
h: H^{2} M \rightarrow \mathscr{F}_{s} .
$$

For any $g \in L_{m}^{2}$ and $\varrho \in H^{2} M . h(\varrho g)=h(\varrho) . \varkappa(g)$.

Now we introduce a structure function $\Theta$ on $\mathscr{F}_{s}$ which enables us to characterize the frames belonging to $h\left(H^{2} M\right)$. For simplicity we use the notation $G_{s}$ for the group Aut $_{s}\left(T T_{0} \mathbb{R}^{m}\right)$ of all $T T$-soldered $\mathscr{D} \mathscr{L}$-automorphisms of $T T_{0} \mathbb{R}^{m}$. Any element $(\varphi, \varphi, \varphi, \sigma) \in G_{s}$ is uniquely expressible in the form $(\varphi, \varphi, \varphi, \sigma)=(\varphi, \varphi, \varphi, b)$. .$(1,1,1, a)$ where $b \in \operatorname{Hom}_{\text {sym }}\left(\mathbb{R}^{m} \times \mathbb{R}^{m}, \mathbb{R}^{m}\right)$, and $a$ is an element of the vector space $\operatorname{Hom}_{\mathrm{ant}}\left(\mathbb{R}^{m} \times \mathbb{R}^{m}, \mathbb{R}^{m}\right)$ of all antisymmetric bilinear maps on $\mathbb{R}^{m}$. Any frame $f \in \mathscr{F}_{s, x}$ is a soldered $\mathscr{L} \mathscr{L}$-isomorphism

$$
f: T T_{0} \mathbb{R}^{m} \rightarrow T T_{x^{M}} M .
$$

Let $\alpha, \alpha^{\prime}: U \subset \mathbb{R}^{m} \rightarrow M$ be local diffeomorphisms with $\alpha(0)=\alpha^{\prime}(0)=x$. The element $\left(T T_{0} \alpha\right)^{-1} f \in G_{s}$ has a unique decomposition

$$
\left(T T_{0} \alpha\right)^{-1} f=g .(1,1,1, a), \quad g \in G_{s s}, \quad a \in \operatorname{Hom}_{\text {ant }}\left(\mathbb{R}^{m} \times \mathbb{R}^{m}, \mathbb{R}^{m}\right) .
$$

Similarly for $\left(T T_{0} \alpha^{\prime}\right)^{-1} f=g^{\prime} .\left(1,1,1, a^{\prime}\right)$. A simple evaluation shows that $g^{\prime}=$ $=\left(T T_{0}\left(\alpha^{\prime-1} \circ \alpha\right)\right) g$, and $a=a^{\prime}$. So we can define a structure function

$\Theta: \mathscr{F}_{s} \rightarrow \operatorname{Hom}_{\text {ant }}\left(\mathbb{R}^{m} \times \mathbb{R}^{m}, \mathbb{R}^{m}\right)$ on the principal fibration $\mathscr{F}_{s}$ of $T T$-soldered frames on TTM by

$$
\Theta(f)=a .
$$


Theorem 3. The structure function $\Theta$ has the following properties:

$\Theta$ is differentiable map.

If $\tilde{g} \in G_{s}$ with the decomposition $\left.\tilde{g}=\tilde{\varphi}, \tilde{\varphi}, \tilde{\varphi}, \tilde{b}\right) \cdot(1,1,1, \tilde{a})$ then $\Theta(f \tilde{g})=\tilde{\varphi}^{-1} \Theta(f)(\tilde{\varphi}, \tilde{\varphi})+\tilde{a}$.

Proof. The verification of (3) is standard, (4) follows by a direct evaluation.

The frames from $\mathscr{F}_{s s}=h\left(H^{2} M\right)$ can be now characterized as follows:

Theorem 4. The frame $f$ belongs to $\mathscr{F}_{\text {ss }}$ if and only if $\Theta(f)=0$.

Now consider the second tangent bundle on $M$ as a double vector fibration $\mathscr{C}=$ $=T T M$ with $\mathscr{A}=\mathscr{B}=\mathscr{V}=T M$, and with projections $p: T T M \rightarrow M, \pi_{1}: T T M \rightarrow$ $\rightarrow T M, \pi_{2}: T T M \rightarrow T M,[11]$. Let $i: T T M \rightarrow T T M$ denote the canonical involution, and denote by $q^{2}$ the projection $q^{2}: \mathscr{F}_{s s} \rightarrow M$.

Lemma 2. Let $z, z^{\prime}$ be elements of TTM, let $\lambda$ be a real number. Then the following is satisfied:

$$
\begin{aligned}
& \text { If } \pi_{1} z=\pi_{1} z^{\prime} \text { then } i\left(z++_{1} z^{\prime}\right)=(i z)+{ }_{2}\left(i z^{\prime}\right) . \\
& \text { If } \pi_{2} z=\pi_{2} z^{\prime} \text { then } i\left(z++_{2} z^{\prime}\right)=(i z)+{ }_{1}\left(i z^{\prime}\right) . \\
& i\left(\lambda_{1} z\right)=\lambda_{\cdot_{2}}(i z), \\
& i\left(\lambda_{\cdot_{2}} z\right)=\lambda_{\cdot_{1}}(i z) .
\end{aligned}
$$

Proof. We shall prove (4). Choose a frame $f \in \mathscr{F}_{s s}=h\left(H^{2} M\right)$ with $q^{2}(f)=$ $=p(z)=p\left(z^{\prime}\right)$. Since $f$ is of the form (2) and $T T_{0} \mathbb{P}^{m}$ is isomorphic to $\mathbb{R}^{3 m}$, there are uniquely determined elements $(a, b, v),\left(a^{\prime}, b^{\prime}, v^{\prime}\right)$ from $\mathbb{R}^{3 m}$ such that

$$
z=f(a, b, v), \quad z^{\prime}=f\left(a^{\prime}, b^{\prime}, v^{\prime}\right) .
$$

Let $i$ be an element of $Z_{s s}\left(\mathbb{R}^{m} \times \mathbb{R}^{m} \times \mathbb{R}^{m}\right)$ determining the canonical involution $i$ on TTM. Here $Z_{\text {ss }}$ denotes the set of all differentiable maps of the given $\mathscr{D} \mathscr{L}$-space into itself commuting with all its strongly $T T$-soldered $\mathscr{D} \mathscr{L}$-automorphisms, [12]. We have

$$
\begin{aligned}
& i\left(z+{ }_{1} z^{\prime}\right)=i f\left(a, b+b^{\prime}, v+v^{\prime}\right)=f\left(\tilde{\imath}\left(a, b+b^{\prime}, v+v^{\prime}\right)\right)= \\
& =f\left(b+b^{\prime}, a, v+v^{\prime}\right)=f(\tilde{\imath}(a, b, v))+{ }_{2} f\left(\tilde{\imath}\left(a, b^{\prime}, v^{\prime}\right)\right)= \\
& =(i z)+{ }_{2}\left(i z^{\prime}\right) .
\end{aligned}
$$

Similarly in the other cases.

Assume a frame $f \in \mathscr{F}$, i.e. a $\mathscr{D} \mathscr{L}$-isomorphism $f: \mathbb{R}^{3 m} \rightarrow T T_{x} M$. It is easily checked that if $\tilde{\imath}: \mathbb{R}^{3 m} \rightarrow T T_{x} M$ is again a $\mathscr{D} \mathscr{L}$-isomorphism. The map

$$
f \rightarrow I f=\text { if } \tilde{\imath}, \quad I: \mathscr{F} \rightarrow \mathscr{F}
$$

will be called the canonical involution on $\mathscr{F}$. 
Theorem 5. The canonical involution I on $\mathscr{F}$ satisfies

$$
\begin{aligned}
& I^{2}=\mathrm{id}, \\
& I\left(\mathscr{F}_{s}\right)=\mathscr{F}_{s}, \\
& \text { If }=f \text { for } f \in \mathscr{F}_{s s} . \\
& \text { If } f \in \mathscr{F}_{s} \text { then } \Theta(I f)=-\Theta f .
\end{aligned}
$$

Proof. (8) is clear. Let $f \in \mathscr{F}_{s}$. Since $\mathscr{F}_{s}$ corresponding to TTM has the group $\operatorname{Aut}_{s}\left(\mathbb{R}^{3 m}\right)=\operatorname{Aut}_{s}\left(T T_{0} \mathbb{R}^{m}\right)=G_{s}$ as its structure group we can choose $\tilde{f} \in \mathscr{F}_{s s}$ and $g=(\varphi, \varphi, \varphi, \sigma) \in G_{s}$ such that $f=\tilde{f} g$. Evaluation of If on an arbitrary $(a, b, v) \in$ $\in \mathbb{R}^{3 m}$ shows that

$$
I f=\tilde{f}(\varphi, \varphi, \varphi, \vec{\sigma})
$$

where $\bar{\sigma}: \mathbb{R}^{m} \times \mathbb{R}^{m} \rightarrow \mathbb{R}^{m}$ is a bilinear map given by $\bar{\sigma}(x, y)=\sigma(y, x)$. Hence $(\varphi, \varphi, \varphi, \bar{\sigma}) \in G_{s}$, and $I f \in \mathscr{F}_{s}$ which proves (9). (10) follows immediately by the equality if $\tilde{\imath}(a, b, v)=f \tilde{\imath}^{2}(a, b, v)$ for $f \in \mathscr{F}_{s s}$. Further, given $f \in \mathscr{F}_{s}$ let $\tilde{f} \in \mathscr{F}_{s s}$ be a unique form with the property $f=\tilde{f}(1,1,1, a)$ where $a \in \operatorname{Hom}_{\text {anc }}\left(\mathbb{R}^{m} \times \mathbb{R}^{m}, \mathbb{R}^{m}\right)$. Evaluation shows that $I f=f(1,1,1,-a)$. By (4) and Theorem 4, $\Theta(f)=\Theta(\tilde{f})+$ $+a=a, \Theta($ If $)=\Theta(\tilde{f})-a=-a$.

Remark. The frames from $\mathscr{F}$ satisfying (10) are also ,soldered" in some sense, but it can be verified that $\mathscr{F}_{s s} \neq\{f \in \mathscr{F}, I f=f\}$.

Theorem 6. Let $\Gamma$ be a $\mathscr{D} \mathscr{L}$-connection on TTM such that the invariant connection

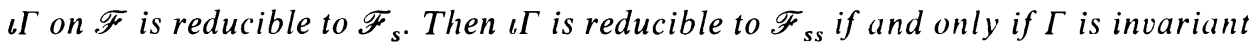
with respect to the canonical involution $i$ on TTM.

Proof. (a) First suppose that $\iota \Gamma$ is reducible to $\mathscr{F}_{s}=h\left(H^{2} M\right)$. Let $z \in T T_{x} M$. Choose a vector $Z \in H_{z}$ from the horizontal space with respect to $\Gamma$ and a frame $f \in h\left(H_{x}^{2} M\right)$. Clearly there exists a unique element $c \in \mathbb{R}^{3 m}$ such that $z=f c$, a value of the map $f$ on $c$ which is an element of the associated fibration TTM determined by an element $f$ of the principal fibration $\mathscr{F}_{s s} \subset \mathscr{F}_{s}$, and an element $c$ of the standard fibre $\mathbb{R}^{3 m}$. Choose a curve $\delta:(-\varepsilon, \varepsilon) \rightarrow M$ such that

$$
\delta(0)=x, \quad\left(\frac{\mathrm{d}}{\mathrm{d} t}\right)_{t=0} \delta(t)=(T p) Z
$$

where $p: T T M \rightarrow M$ is a projection, and consider its horizontal lift with respect to $\iota \Gamma, \gamma:(-\varepsilon, \varepsilon) \rightarrow \mathscr{F}$ with $\gamma(0)=f$. Then

$$
\gamma(0) c=z, \quad\left(\frac{\mathrm{d}}{\mathrm{d} t}\right)_{t=0}(\gamma(t) c)=Z,
$$

and $\gamma(t) \in \mathscr{F}_{s s}$ for $t \in(-\varepsilon, \varepsilon)$ because of the reducibility of $\iota \Gamma$ to $\mathscr{F}_{s s}$. Using again an element $\tilde{i} \in Z_{s s}\left(\mathbb{R}^{3 m}\right)$ and a horizontal curve (with respect to $\left.\Gamma\right) \gamma(t)(\tilde{i} c)$ we obtain (Ti) $Z=(\mathrm{d} / \mathrm{d} t)_{t=0}(\gamma(t)(\tilde{i} c)) \in H_{i z}$, which proves the $i$-invariance.

(b) Now suppose the $i$-invariance of $\Gamma$ on TTM. Let $f \in \mathscr{F}_{s s}$ and $Z \in H_{f}$ where $H_{f}$ 
is horizontal to $\iota \Gamma$. Assume a horizontal curve $\gamma:(-\varepsilon, \varepsilon) \rightarrow \mathscr{F}$ with $\gamma(0)=f$, $(\mathrm{d} / \mathrm{d} t)_{t=0} \gamma(t)=Z$, and choose $\tilde{\gamma}:(-\varepsilon, \varepsilon) \rightarrow \mathscr{F}_{s s}$ such that $q^{2} \tilde{\gamma}=q_{s} \gamma, \tilde{\gamma}(0)=f$. There is a uniquely determined curve $g:(-\varepsilon, \varepsilon) \rightarrow G_{s}$ such that $\gamma(t)=\tilde{\gamma}(t) g(t)$ for $t \in(-\varepsilon, \varepsilon), g(0)=\mathrm{id}_{R^{3 m}}$. For any $c \in \mathbb{R}^{3 m}$, the curve $\gamma c:(-\varepsilon, \varepsilon) \rightarrow T T M$ is horizontal to $\Gamma$. Since $\Gamma$ is $i$-invariant, $i(\gamma c)$ is a horizontal curve, and we have

$$
i(\gamma(t) c)=\tilde{\gamma}(t)(\tilde{i}(g(t) c)) .
$$

Further, $\gamma(\tilde{i} c):(-\varepsilon, \varepsilon) \rightarrow T T M$ is another horizontal curve satisfying $\gamma(t)(\tilde{i} c)=$ $=\tilde{\gamma}(t)(g(t)(\tilde{i c}))$. We have

$$
\begin{aligned}
& p(i(\gamma c))=p(\gamma c)=q^{2} \gamma=p(\gamma(\tilde{i} c)), \\
& i(\gamma(0) c)=\gamma(0)(\tilde{\imath} c) .
\end{aligned}
$$

By the unicity of a horizontal lift, $i(\gamma c)=\gamma(i c)$. By (12), (13) we obtain

$$
\tilde{i}(g(t) c)=g(t)(\tilde{\imath} c) \text { for } t \in(-\varepsilon, \varepsilon) .
$$

Rewriting this equality for components of $g(t)=\left(\varphi_{t}, \varphi_{t}, \varphi_{t}, \sigma_{t}\right)$ and $c=\left(v_{1}, v_{2}, v_{3}\right)$ and comparing them yields $\sigma_{t}\left(v_{1}, v_{2}\right)=\sigma_{t}\left(v_{2}, v_{1}\right)$. Since $c$ was arbitrary we obtain $\sigma_{t} \in \operatorname{Hom}_{\mathrm{sym}}\left(\mathbb{R}^{m} \times \mathbb{R}^{m}, \mathbb{R}^{m}\right)$ for $t \in(-\varepsilon, \varepsilon)$. Consequently, $g(t) \in G_{s s}$ for $t \in(-\varepsilon, \varepsilon)$. Hence $\gamma(t)=\tilde{\gamma}(t) g(t) \in h\left(H^{2} M\right)$ and

$$
Z=\left(\frac{\mathrm{d}}{\mathrm{d} t}\right)_{t=0} \gamma(t) \in T_{f}\left(\mathscr{F}_{s s}\right),
$$

which proves the reducibility of $\iota \Gamma$ to $\mathscr{F}_{s s}$.

In the case of the functor $T T^{*}$, similar statements can be proved. Let $\widetilde{\mathscr{F}}$ or $\widetilde{\mathscr{F}}_{\text {s }}$ denote the fibration of frames or of $T T^{*}$-soldered frames, respectively, on $T T^{*} M$. We introduce a morphism

$$
\tilde{h}: H^{2} M \rightarrow \tilde{\mathscr{F}}
$$

similarly as above. For $\varrho=j_{0}^{2} \alpha \in H_{x}^{2} M, \alpha(0)=x$, define $\tilde{h}(\varrho)=T T_{0}^{*} \alpha^{-1}: T T_{0}^{*} \mathbb{R}^{m} \rightarrow$ $\rightarrow T T_{x}^{*} M$. This definition depends only on the 2 -jet of the local diffeomorphism $\alpha: U \subset \mathbb{R}^{m} \rightarrow M$, and since $T T_{0}^{*} \alpha$ respects the natural soldering, $\tilde{h}$ takes its values in $\widetilde{\mathscr{F}}_{s}$. Again, $\tilde{h}: H^{2} M \rightarrow \widetilde{\mathscr{F}}_{s}$ is a monomorphism of principal fibrations, $\tilde{h}(\varrho g)=$ $=\tilde{h}(\varrho) \tilde{\varkappa}(g)$ for $\varrho \in H^{2} M, g \in L_{m}^{2}$. Here $\tilde{\varkappa}: L_{m}^{2} \rightarrow \operatorname{Aut}_{s}\left(T T_{0}^{*} \mathbb{R}^{m}\right)$ is an isomorphism identifying this both groups given by

$$
\tilde{\chi}(\varphi, \sigma)=\left(\varphi^{*-1}, \varphi, \varphi^{*-1}, \psi\right),
$$

where a bilinear map $\psi$ is defined by the equality

$$
\left\langle\varphi^{-1} \sigma\left(v_{1}, \varphi^{-1}\left(v_{2}\right), a\right\rangle=-\left\langle v_{2}, \psi\left(a, v_{1}\right)\right\rangle .\right.
$$

Let $\widetilde{G}_{s}$ (or $\widetilde{G}_{s s}$ ) denote the group of all $T T^{*}$-soldered (or strongly $T T^{*}$-soldered, respectively) $\mathscr{D} \mathscr{L}$-automorphisms of $T T_{0}^{*} \mathbb{R}^{m},[13]$.

Lemma 3. Any element $\left(\varphi^{*-1}, \varphi, \varphi^{*-1}, \psi\right) \in \widetilde{G}_{s}$ has a unique decomposition

$$
\left(\varphi^{*-1}, \varphi, \varphi^{*-1}, \psi\right)=\left(\varphi^{*-1}, \varphi, \varphi^{*-1}, \varepsilon\right)\left(\mathrm{id}^{*-1}, \mathrm{id}, \mathrm{id}^{*-1}, \beta\right)
$$


where $\varepsilon: \mathbb{R}^{m *} \times \mathbb{R}^{m} \rightarrow \mathbb{R}^{m *}$ is a $\varphi$-symmetric bilinear map, and $\beta: \mathbb{R}^{m *} \times \mathbb{R}^{m} \rightarrow$ $\rightarrow \mathbb{R}^{m *}$ is an id-antisymmetric bilinear map.

Remark. In our case, $\varepsilon$ is $\varphi$-symmetric ( $\varphi$-antisymmetric) if

$$
\begin{aligned}
& \left\langle v, \varepsilon\left(a, \varphi^{-1}(w)\right\rangle=\left\langle w, \varepsilon\left(a, \varphi^{-1}(v)\right\rangle\left(\text { or } \left\langlev, \varepsilon\left(a, \varphi^{-1}(w)=\right.\right.\right.\right.\right. \\
& =-\left\langle w, \varepsilon\left(a, \varphi^{-1}(v)\right\rangle\right) \text { for } \quad v, w \in \mathbb{R}^{m}, \quad a \in \mathbb{R}^{m *} .
\end{aligned}
$$

Proof. The unicity of the decomposition is based on the fact that both the $\varphi$ symmetric and $\varphi$-antisymmetric parts of the bilinear map $\psi=\varepsilon+\varphi^{*-1} \beta$ are determined uniquely. Let us prove the existence. Define a bilinear $\sigma: \mathbb{R}^{m} \times \mathbb{R}^{m} \rightarrow$ $\rightarrow \mathbb{R}^{m}$ by

$$
\left\langle\varphi^{-1} \sigma\left(v_{1}, \varphi^{-1}\left(v_{2}\right)\right), a\right\rangle=-\left\langle v_{2}, \psi\left(a, v_{1}\right)\right\rangle,
$$

and denote by $\sigma^{\prime}, \sigma^{\prime \prime}$ its symmetric or antisymmetric parts, tespectively. Further, let bilinear maps $\varepsilon, \tilde{\beta}: \mathbb{R}^{m *} \times \mathbb{R}^{m} \rightarrow \mathbb{R}^{m *}$ be introduced by

$$
\begin{aligned}
& \left\langle\varphi^{-1} \sigma^{\prime}\left(v_{1}, \varphi^{-1}\left(v_{2}\right), a\right\rangle=-\left\langle v_{2}, \varepsilon\left(a, v_{1}\right)\right\rangle,\right. \\
& \left\langle\varphi^{-1} \sigma^{\prime \prime}\left(v_{1}, \varphi^{-1}\left(v_{2}\right)\right), a\right\rangle=-\left\langle v_{2}, \tilde{\beta}\left(a, v_{1}\right)\right\rangle .
\end{aligned}
$$

It can be checked that $\varepsilon$ is $\varphi$-symmetric, $\tilde{\beta}$ is $\varphi$-antisymmetric, $\beta=\varphi^{*} \tilde{\beta}$ is id-antisymmetric, and

Further,

$$
\left(\varphi^{*-1}, \varphi, \varphi^{*-1}, \varepsilon\right)\left(\mathrm{id}^{*-1}, \mathrm{id}, \mathrm{id}^{*-1}, \beta\right)=\left(\varphi^{*-1}, \varphi, \varphi^{*-1}, \varepsilon+\tilde{\beta}\right) .
$$

$$
\begin{aligned}
& -\left\langle v_{2},(\varepsilon+\beta)\left(a, v_{1}\right)\right\rangle= \\
& =\left\langle\varphi^{-1} \sigma^{\prime}\left(v_{1}, \varphi^{-1}\left(v_{2}\right)\right), a\right\rangle+\left\langle\varphi^{-1} \sigma^{\prime \prime}\left(v_{1}, \varphi^{-1}\left(v_{2}\right), a\right\rangle=\right. \\
& =-\left\langle v_{2}, \psi\left(a, v_{1}\right)\right\rangle .
\end{aligned}
$$

Therefore $\varepsilon+\beta=\psi$, which proves the existence.

Now we shall describe frames from $\tilde{h}\left(H^{2} M\right)=\tilde{\mathscr{F}}_{\text {ss }}$ by means of a structure function

$$
\tilde{\Theta}: \tilde{\mathscr{F}}_{s} \rightarrow \operatorname{Hom}_{\text {ant }}\left(\mathbb{R}^{m *} \times \mathbb{R}^{m}, \mathbb{R}^{m *}\right) .
$$

Let $f \in \widetilde{\mathscr{F}}_{s, x}$, and let $\alpha \in U \subset \mathbb{R}^{m} \rightarrow M$ be a local diffeomorphism with $x(0)=x$. Then $\left(T T_{0}^{*} \alpha^{-1}\right) f \in \tilde{G}_{s}$, and there is a unique decomposition $\left(T T_{0}^{*} \alpha^{-1}\right) f=$ $=g\left(\mathrm{id}^{*-1}, \mathrm{id}, \mathrm{id}^{*-1}, \beta\right)$ where $g \in \widetilde{G}_{s s}$, and an id-antisymmetric $\beta \in \operatorname{Hom}_{\text {ant }}\left(\mathbb{R}^{m *} \times\right.$ $\left.\times \mathbb{R}^{m}, \mathbb{R}^{m *}\right)$ is independent of the choice of $\alpha$ with the above property. Hence we can put

$$
\widetilde{\Theta}(f)=\beta \text {. }
$$

Theorem 7. The structure function $\widetilde{\Theta}$ has the properties

$\widetilde{\Theta}$ is differentiable.

If $\tilde{g} \in \widetilde{G}_{s}$ with the decomposition $\tilde{g}=\left(\tilde{\varphi}^{*-1}, \tilde{\varphi}, \tilde{\varphi}^{*-1}, \tilde{b}\right)$.

$$
\begin{aligned}
& .\left(\mathrm{id}^{*-1}, \mathrm{id}, \mathrm{id}^{*-1}, \tilde{\beta}\right) \text { then } \\
& \tilde{\Theta}(f \tilde{g})=\tilde{\varphi}^{*} \tilde{\Theta}(f)\left(\tilde{\varphi}^{*-1}, \tilde{\varphi}\right)+\tilde{\beta} .
\end{aligned}
$$

The proof uses similar arguments as the proof of Theorem 3. 
Theorem 8. $f \in \tilde{h}\left(H^{2} M\right)$ if and only if $\widetilde{\Theta}(f)=0$.

The following lemma can be proved.

Lemma 4. $A$ map $\mu: G_{s} \rightarrow \widetilde{G}_{s}$ given by the formula

$$
\mu(\varphi, \varphi, \varphi, \sigma)=\left(\varphi^{*-1}, \varphi, \varphi^{*-1}, \varepsilon\right)
$$

where $:: \mathbb{R}^{m *} \times \mathbb{R}^{m} \rightarrow \mathbb{R}^{m *}$ is a bilinear map given $b y$

$$
\begin{aligned}
& \left\langle\varphi^{-1} \sigma\left(v_{1}, \varphi^{-1}\left(v_{2}\right)\right), a\right\rangle=-\left\langle v_{2}, \varepsilon\left(a, v_{1}\right)\right\rangle \text { for } v_{1}, v_{2} \in \mathbb{R}^{m}, \\
& a \in \mathbb{R}^{m *}
\end{aligned}
$$

is a group isomorphism. Moreover, $\mu$ maps the subgroup $G_{s s} \subset G_{s}$ isomorphically onto $\tilde{G}_{s s} \subset \tilde{G}_{s}$.

A map $\tilde{h} h^{-1}: \tilde{\mathscr{F}}_{s s} \rightarrow \widetilde{\mathscr{F}}_{s s}$ is an isomorphism of principal fibrations over a structure group morphism $\mu \mid G_{s s}: G_{s s} \rightarrow \tilde{G}_{s s}$. We will construct an extension of $\tilde{h} h^{-1}$ as follows. Let $f \in \mathscr{F}_{s . x}$, and choose $f_{0} \in \mathscr{F}_{s s . x}$. Then there is a single element $\hat{g} \in G_{s}$ such that $f=f_{0} \hat{g}$. Define

$$
\varkappa(f)=\left(\tilde{h} h^{-1}\left(f_{0}\right)\right) \mu(\hat{g}) .
$$

It can be verified that this definition is independent of the choice of $f_{0}$. For $f \in \mathscr{F}_{s s}$, we have $x(f)=\tilde{h} h^{-1}(f)$. So we have proved

Theorem 9. The map $x: \mathscr{F}_{s} \rightarrow \widetilde{\mathscr{F}}_{s}$ is an isomorphism of principal fibrations over the structure group isomorphism $\mu: G_{s} \rightarrow \widetilde{G}_{s}$, and $\varkappa$ maps a principal subfibration $\widetilde{F}_{\text {ss }}$ onto $\widetilde{\mathscr{H}}_{s .}$.

Invariant connections $\Gamma$ on $\mathscr{F}_{s}$ and $\tilde{\Gamma}$ on $\tilde{\mathscr{F}}_{s}$ will be called conjugated if $(T \varkappa) \Gamma=$ $=\tilde{\Gamma}$. The existence of a conjugated connection to a given connection on $\mathscr{F}_{s}$ or $\widetilde{\mathscr{F}}_{s}$, respectively, is clear.

Theorem 10. Let $\tilde{\Gamma}$ be an invariant connection on $\tilde{\mathscr{F}}_{s}$. Then $\tilde{\Gamma}$ is reducible onto $\tilde{h}\left(H^{2} M\right)$ if and only if the corresponding conjugated connection $\Gamma$ is reducible to $h\left(H^{2} M\right)$.

\section{References}

[1] J. Janı̌̌ka: Geometrical properties of prolongation functors, Časop. pěst. mat. 110 (1985), $77-86$.

[2] I. Kolär: On the jet prolongation of smooth categories, Bull. Acad. Polon., Math., astr. et phys. Vol. $X X I V$, No. 10 (1976), 883-887.

[3] I. Kolár: On the second order tangent bundle and generalized Lie derivatives, Tensor, N.S. $38(1982), 98-102$.

[4] I. Kolár, Z. Radziszewski: Natural transformations of second order tangent and cotangent functors, Czech. Mat. J. 38 (113) 1988, 274-271.

[5] L. Mangiarotti, M. Modugno: Fibred spaces, jet spaces and connections in field theories, Proceed. of the meeting "Geometry and Physics", Florence 1987.

[6] J. Pradines: Répresentation des jets non holonomes par des morphismes vectoriels doubles soudés, C.R. Acad. Sci. Paris Sér. A, 278 (1974), 1523-1526. 
[7] J. Pradines: Fibres vectoriels double symétriques et des jets holonomes d'ordre 2, C.R. Acad. Sci. Paris Sér. A, 278 (1974), 1557-1560.

[8] A. Vanžurová: Connections on the second tangent bundle, Časop. pěst. mat. 108 (1983), $258-264$.

[9] A. Vanžurová: On geometry of the third tangent bundle, Acta UPO, Fac, rer. nat. 82 (1985), $81-96$.

[10] A. Vanžurová: Double vector spaces, Acta UPO, Fac. rer. nat. 88 (1987), Math. $X X I I$, 9-25.

[11] A. Vanžurová: Double linear connections, Acta UPO, Fac. rer. nat. (990), to appear.

[12] A. Vanžurová: Natural transformations of the second tangent functor and soldered morphisms, Acta UPO, Fac. rer. nat. (991), to appear.

[13] A. Vanžurová: Soldered double linear morphisms, to appear.

Author's address: 77146 Svobody 26, Olomouc, Czechoslovakia (Katedra algebry a geometrie PU). 